\title{
Non-Cesarean Uterine Surgical Scarring
}

National Cancer Institute

\section{Source}

National Cancer Institute. Non-Cesarean Uterine Surgical Scarring. NCI Thesaurus. Code C112856.

A scar/healing of the myometrium that occurs after uterine injury or after non-cesarean surgery. 